\title{
Molecular Targeted Therapy of Lung Cancer: EGFR Mutations and Response to EGFR Inhibitors
}

\author{
D.A. Haber, D.W. Bell, R. Sordella, E.L. KwaK, N. Godin-Heymann, \\ S.V. SHARMA, T.J. LYNCH, AND J. SETTLEMAN \\ Massachusetts General Hospital Cancer Center and Harvard Medical School, \\ Charlestown, Massachusetts 02129
}

\begin{abstract}
Somatic mutations within the kinase domain of the epidermal growth factor receptor (EGFR) are present in approximately $10 \%$ of non-small-cell lung cancer (NSCLC), with an increased frequency in adenocarcinomas arising in nonsmokers, women, and individuals of Asian ethnicity. These mutations lead to altered downstream signaling by the receptor and appear to define a subset of NSCLC characterized by "oncogene addiction" to the EGFR pathway, which displays dramatic responses to the reversible tyrosine kinase inhibitors gefitinib and erlotinib. The rapid acquisition of drug resistance in most cases, either through mutation of the "gateway" residue in the EGFR kinase domain or by alternative mechanisms, appears to limit the impact on patient survival. Irreversible inhibitors of EGFR display continued effectiveness in vitro against cells with acquired resistance and are now undergoing genotype-directed clinical trials. The molecular and clinical insights derived from targeting EGFR in NSCLC offer important lessons for the broader application of targeted therapeutic agents in solid tumors.
\end{abstract}

Lung cancer remains the leading cause of cancerrelated deaths in the U.S., and although advances in chemotherapy for advanced disease have resulted in improved survival, the duration of responses is limited and the associated toxicity is high. The advent of molecular targeted therapy, specifically the development of smallmolecule inhibitors of the epidermal growth factor receptor (EGFR), has recently provided new therapeutic options for the most common lung tumor histology, non-small-cell lung cancer (NSCLC). These orally administered agents, gefitinib (Iressa) and erlotinib (Tarceva), are well tolerated and have been shown to have dramatic efficacy in a subset of NSCLC. Here we discuss the finding that activating somatic mutations in EGFR appear to define a biological subset of NSCLC susceptible to inhibition of this pathway.

\section{CLINICAL TRIALS OF GEFITINIB IN NSCLC}

Gefitinib was the first small-molecule inhibitor of EGFR developed for clinical application (Wakeling et al. 2002). It was modeled after imatinib (Gleevec), which inhibits the ABL, C-KIT, and platelet-derived growth factor receptor (PDGFR) kinases, and is effective in the treatment of chronic myeloid leukemia (CML) and gastrointestinal stromal tumor (GIST). Like imatinib, gefitinib and erlotinib, the two clinically approved agents targeting EGFR, act as competitive (i.e., reversible) inhibitors for binding to the ATP pocket of the kinase. Despite the expression of EGFR in most epithelial cancers, initial clinical trials of gefitinib were discouraging, with the exception of NSCLC, where it was shown to induce partial responses (PR) in approximately $10 \%$ of cases (Kris et al. 2003). These responses were notable in that they were exceptionally rapid and profound, and they were more frequently observed in cases with specific clinical characteristics: adenocarci- nomas (often with bronchioalveolar differentiation), and tumors arising in nonsmokers, women, and patients of East Asian ethnic background (Table 1). Whereas the average duration of a PR was 6-7 months before the disease recurred, rare patients had sustained responses lasting up to 3 years. Our own interest in exploring the genetic basis for this dramatic difference in drug response was triggered by such a case.

\section{EGFR MUTATIONS IN GEFITINIB- RESPONSIVE NSCLC}

By analogy with the imatinib-responsive diseases CML and GIST, which harbor the BCR-ABL translocation or activating C-KIT mutations, respectively, we reasoned that cases of NSCLC with dramatic responses to gefitinib might harbor genetic alterations affecting the target receptor, EGFR. EGFR overexpression was not

Table 1. Clinical Predictors of Gefitinib Responses in Lung Cancer (NSCLC)

\begin{tabular}{ll}
\hline Overall response rate & $10-20 \%$ \\
Median duration of response & $\begin{array}{c}7 \text { months (range } 4.4 \\
\text { to }>18.6 \text { ) }\end{array}$ \\
$\begin{array}{l}\text { Clinical predictors of response } \\
\text { nonsmokers }\end{array}$ & $29.4 \%$ \\
smokers & $4.6 \%$ \\
female & $17.5 \%$ \\
male & $5.1 \%$ \\
Japan & $20 \%$ \\
U.S./Europe & $10 \%$ \\
histology & adenocarcinoma \\
& (bronchioalveolar) \\
& $>$ others \\
\hline
\end{tabular}

Adapted from FDA Approval Summary: Clinical Cancer Research (2004) 10: 1212. 
linked to gefitinib response in clinical trials, nor did we find evidence of specific in-frame deletions affecting the EGFR extracellular domain, which had been previously reported in glioblastomas (so-called EGFR vIII mutation). Instead, we observed point mutations and small inframe deletions within the intracellular kinase domain of EGFR (Lynch et al. 2004). Initial cases available for analysis were derived from 275 patients with chemotherapyrefractory NSCLC, who had been treated with single agent gefitinib over a 3-year period at Massachusetts General Hospital. Of these, 25 patients $(9 \%)$ were noted to have a dramatic response to gefitinib, and tumor specimens were available for analysis from nine of these cases. Of note, all tumor specimens had been collected at the time of initial diagnosis, typically preceding multiple rounds of chemotherapy treatment, and eventual therapy with gefitinib. Eight of the nine gefitinib-responsive tumors were found to harbor an EGFR kinase domain mutation, whereas none of seven nonresponders had a mutation $(p<0.001)$ (Fig. 1). Coincident with our report, another group from Dana Farber Cancer Institute described EGFR mutations in all five patients with gefitinib-responsive NSCLC (Paez et al. 2004). A third group at Memorial Sloan Kettering Cancer Center described a similar frequency of EGFR mutations in patients with both gefitinib- and erlotinib-responsive NSCLC (Pao et al. 2005). From these reports and subsequent reports from additional research laboratories, it appears that approximately $80 \%$ of NSCLC cases identified on the basis of dramatic responses to EGFR inhibitors harbor such mutations, compared with $6 \%$ of nonresponders. The overall frequency of EGFR kinase domain mutations in unselected cases of NSCLC is approximately $10 \%$ in the U.S. and Europe. Remarkably, the frequency of these mutations is increased in cases with adenocarcinoma (especially adenocarcinomas with areas of bronchioalveolar differentiation), in women, nonsmokers, and patients of Asian origin - consistent with the clinically determined indicators of drug-responsive cancers.

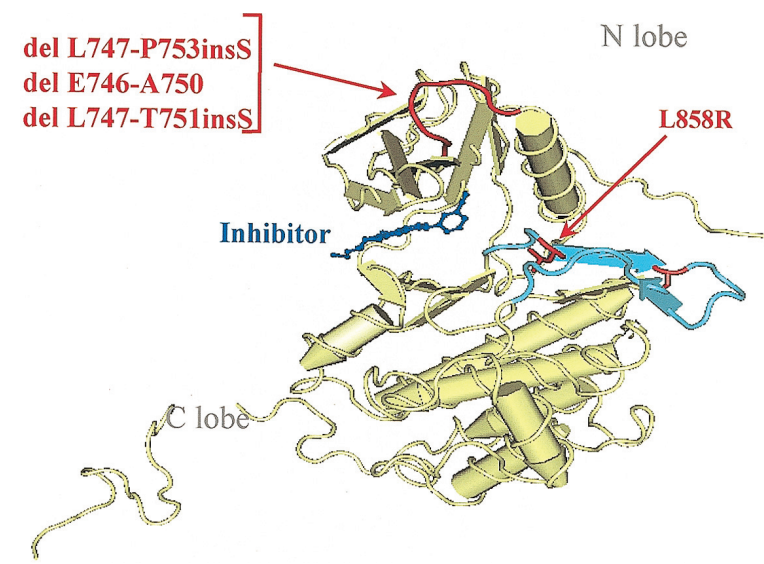

Figure 1. Representative structure of the EGFR ATP pocket, denoting the position of the recurrent in-frame nested deletions and the L858R missense mutation (shown in red), which together comprise $\sim 90 \%$ of activating mutations associated with gefitinib responsiveness. The inhibitor is shown in blue. (Adapted from Lynch et al. 2004.)

\section{PREDICTIVE VALUE OF EGFR MUTATIONS}

Although the majority of NSCLC identified by virtue of a dramatic clinical response to gefitinib or erlotinib harbor EGFR mutations, not all cases with such mutations demonstrate a potent drug response. To address the predictive value of EGFR mutations, we analyzed clinical specimens collected as part of the original "IDEAL" clinical trials, in which gefitinib was administered to chemotherapy-refractory NSCLC patients, and which led to the first identification of subsets with dramatic responses and specific clinical characteristics. As is common with such retrospective analyses, only a fraction of tumor specimens were recovered, and only a subset of those contained sufficient tumor material for molecular analysis. Nonetheless, we were able to analyze approximately $30 \%$ of the cases in these large international trials and to confirm that clinical characteristics of cases analyzed were comparable with those of the entire cohort (Bell et al. 2005). Gefitinib responses were found in $46 \%$ of EGFR mutant NSCLC, compared with $10 \%$ of cases with wild-type EGFR $(p=0.005)$. Most responses were transient ( $\sim 7$ months' duration), and no survival difference was observed between responsive and nonresponsive cases. These results are consistent with other reports (Han et al. 2005; Mitsudomi et al. 2005) that, in aggregate, identify drug responses in $40-80 \%$ of EGFR mutant cases. Our results differ from a recent publication in which only $16 \%$ of EGFR mutant tumors were found to respond to erlotinib (Tsao et al. 2005), but that study included large numbers of novel unconfirmed sequence variants identified in a "single pass" sequencing of PCR products. In contrast, EGFR mutations identified in drug responders constitute a small number of well-defined recurrent mutations within the kinase domain (see below); we and other investigators have found that PCR amplification of EGFR from small amounts of formalin-fixed, paraffin-embedded specimens can result in a significant number of sequence variants which are not reproducible in duplicate analysis, and can therefore be considered PCR artifacts. Such findings highlight the importance of establishing uniformly high quality DNA sequencing methods for analyzing tumor specimens in order to derive a consensus conclusion regarding the relationship between tumor genotypes and clinical outcomes across multiple clinical centers.

Nonetheless, it is evident that not all NSCLC with EGFR kinase mutations are responsive to treatment with EGFR inhibitors. It is possible that additional genetic lesions in nonresponsive cases may have relieved the "dependence" of these EGFR-mutant tumors on signaling through the EGFR pathway. Supporting that hypothesis is the recent observation that absence of immunoreactivity for PTEN protein expression may identify brain tumors with the EGFR vIII mutation that progress on erlotinib, whereas those expressing wild-type PTEN together with EGFR vIII show a modest response (Mellinghoff et al. 2005). PTEN suppresses the AKT survival pathway, a key effector of EGFR signaling, and hence, PTEN inactivation might compensate for reduced EGFR signaling. However, in our analysis of NSCLC cases with EGFR kinase mutations that did not respond to gefitinib, we did not observe an increased frequency of PTEN mutations, 
nor did we detect mutations in other pathways that modulate EGFR signaling or general apoptotic signals (Bell et al. 2005). Modulators of gefitinib response in EGFR mutant NSCLC thus remain to be identified. Another critical consideration is that retrospective analyses typically involve only a fraction of tumor specimens collected at the time of initial diagnosis, whereas gefitinib or erlotinib therapy is administered up to 1-2 years later, following multiple courses of chemotherapy. The true predictive value of EGFR mutations will need to be assessed in prospective clinical trials, in which EGFR status is assessed at the time of drug therapy.

The dramatic responses to gefitinib and erlotinib observed in a subset of NSCLC have not been associated with a commensurate improvement in overall survival for the cohort of patients treated with these agents. For erlotinib, but not gefitinib, a moderate improvement in overall survival for all treated patients appears to be driven by disease stabilization in a larger fraction of cases, not specifically by the subset of cases with EGFR mutations and dramatic responses (Shepherd et al. 2005). Although subtle pharmacological differences between these drugs or differences in the composition of clinical cohorts may have contributed to this difference between the two tyrosine kinase inhibitors, this distinction may also have resulted from the higher dosing level chosen for erlotinib in these clinical trials.

Given the relatively high prevalence of EGFR mutations in NSCLC in East Asia, a number of trials conducted in Korea and Japan have provided the most compelling data for a survival advantage (up to 2 years) in EGFR-mutant cases treated with gefitinib (Han et al. 2005; Mitsudomi et al. 2005). In U.S. and European trials, no survival difference was evident in the relatively smaller subset of EGFR mutant cases, despite an increased drug response in such cases. This may reflect the relatively rapid acquisition of drug resistance in many cases with initially responsive disease (see below).

\section{EGFR MUTATIONS AND EGFR AMPLIFICATION}

Amplification of the EGFR gene is relatively common in malignant gliomas, where it is often associated with the EGFR vIII mutation. Despite the frequency of this genetic abnormality in EGFR, gliomas exhibit very modest responses to either gefitinib or erlotinib. These observations suggest either that gliomas are less dependent than NSCLC on EGFR signaling, or alternatively, that kinase mutations lead to qualitative differences in these signals, which underlie much of the drug response in NSCLC (see below). In NSCLC, amplification of EGFR, involving either wild-type alleles or alleles harboring a kinase mutation, has also been reported. Some studies have used FISH analysis to grade tumors as having a range of abnormalities, from low-grade nonspecific aneuploidy to high levels of specific EGFR amplification, leading to the conclusion that high-level aneuploidy and/or EGFR amplification, together with increased EGFR protein expression measured by immunohistochemistry, provide a predictive index of susceptibility to EGFR inhibitors (Hirsch et al. 2005). In our analysis of the IDEAL clinical trials, we measured EGFR amplification by quantitative PCR (qPCR), using control probes selected to exclude aneuploidy (Bell et al. 2005). Only 7\% of NSCLC were found to have significant EGFR amplification using this approach, compared with $\sim 30 \%$ reported using FISH analysis, presumably reflecting the lower frequency of highlevel amplification measured across the entire tumor specimen, compared with individual cell analysis using FISH. Most EGFR amplification detected by qPCR reflected amplification of the wild-type allele, although some cases had amplification of a mutant allele. EGFR amplification as measured by qPCR was predictive of gefitinib responsiveness, although not as strongly as EGFR mutations. Remarkably, however, the characteristics of NSCLC with EGFR amplification differed markedly from those with kinase domain mutations (Table 2). EGFR mutations were more common in adenocarcinomas and tumors arising in women and nonsmokers, all of which are characteristics previously identified in NSCLC tumors with dramatic responses to this agent. In striking contrast, tumors with EGFR amplification were indistinguishable from the entire cohort, being more common in men and in smokers, and not being associated with a specific histological type (Bell et al. 2005). Furthermore, EGFR mutations were more commonly observed in tumors from younger patients, whereas amplification predominated in tumors from older patients. Taken together, these observations suggest that although both markers may denote alterations in EGFR signaling that are directly relevant to tumor growth and drug response, they appear to arise in distinct subsets of NSCLC. Additional studies are needed in which tumor samples are prospectively collected at the time of drug treatment for a meaningful comparison of EGFR mutations and amplification (or both) as predictors of drug response.

Table 2. EGFR Mutations and Amplification Define Distinct Patient Subgroups

\begin{tabular}{lcccc}
\hline Characteristics & $\begin{array}{c}\text { Mutation } \\
\text { frequency }(\%)\end{array}$ & $p$ value & $\begin{array}{c}\text { Amplification } \\
\text { frequency }(\%)\end{array}$ & $p$ value \\
\hline Nonsmoker & 25.5 & 0.0004 & 6.3 & 0.82 \\
& 7.7 & & 8.1 & \\
Adenocarcinoma/BAC & 17.4 & 0.0001 & 6.9 & 0.74 \\
& 5.1 & & 7.8 & \\
Age $<64$ years & 14.3 & 0.031 & 6.6 & 0.0009 \\
$\quad>64$ years & 6.8 & & 18.5 & \\
\hline
\end{tabular}

Adapted from Bell et al. 2005. 


\section{FUNCTIONAL PROPERTIES OF MUTANT EGFR}

The EGFR kinase domain mutations identified in gefitinib-responsive NSCLC are clustered around the ATPbinding pocket, which constitutes the drug-binding site (Fig. 1) (Lynch et al. 2004; Paez et al. 2004; Pao et al. 2005). Large-scale tumor genotyping studies have now shown that approximately $80-90 \%$ of mutations consist either of small, nested in-frame deletions, centered around the LREA motif in the kinase domain, or the L858R missense mutation in the activation loop. In comparing NSCLC tumor cell lines with either a deletion mutation or L858R with comparable NSCLC cell lines expressing wild-type EGFR, we observed that cells with mutant EGFR displayed increased proliferation when cultured in the absence of serum but in the presence of exogenous EGF (Sordella et al. 2004). Thus, these mutations may mediate ligand-dependent proliferation. Consistent with this model, immunohistochemical analysis of primary NSCLCs with mutant EGFR shows expression of its two ligands, EGF and TGF- $\alpha$, in tumor cells themselves, but not in the reactive stroma (Riemenschneider et al. 2005). Taken together, these observations suggest that somatic EGFR mutations arise in NSCLC cells that express EGFR ligands, thus enhancing a potential autocrine growth loop.

To dissect the effect of EGFR mutations on downstream signaling pathways, we undertook a series of experiments, including transient transfection of EGFR-expression plasmids into Cos-7 cells, which express low levels of endogenous EGFR; stable transfection of these plasmids into nontransformed mouse mammary epithelial cells, expression of endogenous erb-B family members; and comparison of established lung carcinoma cell lines of bronchioalveolar histology, either expressing wild-type EGFR or the in-frame deletion or L858R mutants (Sordella et al. 2004). Our findings were consistent across these different cell types and are summarized as follows: (1) Neither EGFR mutation enhances ligand-independent EGFR signaling; however, they lead to a 2- to 3 -fold increase in both the magnitude and duration of EGFR autophosphorylation following exposure to the ligand EGF. (2) The pattern of EGFR autophosphorylation differs between wild-type and mutant receptors, with Tyr-992 and Tyr-1068 phosphorylation specifically increased in the mutants. EGFR Tyr-845 is specifically autophosphorylated to high levels by L858R, but not the deletion mutant. The total level of receptor autophosphorylation is unaltered between wild type and mutant, suggesting the importance of these qualitative differences in ligand-mediated activation. (3) Activation of mutant EGFR selectively enhances activation of downstream pathways. Specifically, activation of AKT and STAT pathways is dramatically increased following phosphorylation of the mutant receptors, whereas activation of the ERK pathway is, if anything, reduced compared with the wild-type EGFR (Sordella et al. 2004). Taken together, these observations suggest that mutant EGFRs selectively activate survival or antiapoptotic pathways (STAT, AKT), whereas the wild-type receptor is relatively more potent in triggering proliferative signals (ERK) (Fig. 2).

\section{ONCOGENE ADDICTION AND GEFITINIB RESPONSE}

The signaling differences evident between wild-type and mutant EGFR are correlated with enhanced sensitivity to tyrosine kinase inhibition. Treatment of some NSCLC cell lines harboring mutant EGFR with gefitinib demonstrates a 50- to 100-fold increase in drug sensitiv-

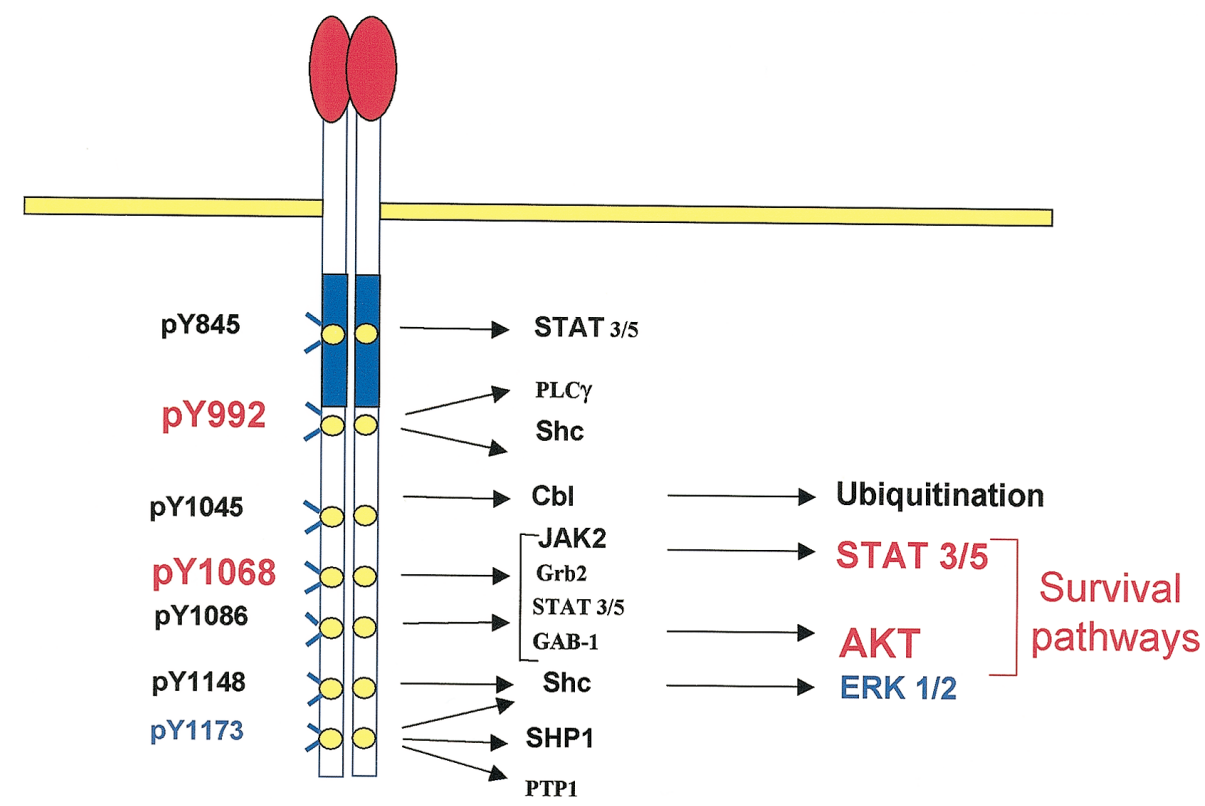

Figure 2. Schematic representation of EGFR-dependent signaling pathways. EGFR tyrosine residues shown in red are preferentially autophosphorylated following EGF stimulation of the mutant receptors. The AKT and STAT pathways are preferentially activated by the mutant receptors. (Adapted from Sordella et al. 2004.) 
ity, compared with cell lines expressing the wild-type receptor. More significantly, the importance of mutant EGFR signaling can be demonstrated using siRNA strategies. Thus, in NSCLC cells with wild-type EGFR, knockdown of the receptor using siRNA has minimal impact on cellular proliferation. In contrast, in cells with mutant EGFR, a comparable knockdown triggers widespread apoptosis (Sordella et al. 2004). Furthermore, in cells expressing the deletion mutation, transfection of an siRNA construct specifically targeting this mutant mRNA triggers cell death, whereas in comparable cells with the L858R mutation, siRNA targeting that specific missense mutation has the same effect. These observations suggest a model in which expression of the mutant allele is itself required for cell survival, with apoptosis initiated following withdrawal of these survival signals, either following treatment with gefitinib or EGFR siRNA. Such a scenario has been proposed to underlie oncogene addiction, a phenomenon according to which cancer cells harboring multiple genetic lesions may nonetheless display exquisite dependency on a single oncogenic stimulus, in whose absence they undergo cell death or growth arrest (Weinstein 2002). On the basis of data using temperature-sensitive oncogene mutants, we have proposed that cells need not be dependent on a persistent oncogenic stimulus per se, but rather that the abrupt withdrawal of signaling from such a critical pathway may lead to a transient imbalance between downstream apoptotic and antiapoptotic signals, thus triggering rapid cell death. We refer to this mechanism as "oncogenic shock" (Sharma et al. 2006). Experimental validation of the oncogenic shock model will be important in a number of model systems, since it predicts that following the transient signaling imbalance which arises upon acute inactivation of an oncogene, cancers may adapt to loss of the oncogenic signal and thus acquire independence from the oncogene. As drug combination regimens emerge, involving concomitant use of targeted and classic chemotherapeutic agents, understanding the mechanistic basis of oncogene addiction will be essential to ensuring an enhancement, rather than a suppression, of drug response.

\section{ENHANCED INHIBITION OF MUTANT EGFR BY GEFITINIB}

The arguments presented above support a biological dependence mechanism by which the mutant EGFR identifies a tumor in which EGFR signals are critical for cell survival. In addition to this mechanism, we have also observed that mutant EGFRs display an approximately 10fold increased sensitivity to gefitinib-mediated inhibition. This is evident in transiently transfected Cos-7 cells, as well as in cell lines stably expressing wild-type or mutant EGFR (Lynch et al. 2004). Although it is difficult to extrapolate such in vitro results to the clinical setting, it is of interest that the measured trough plasma concentrations achievable at clinically recommended gefitinib dosage are predicted to completely abrogate signaling by mutant EGFR, but not by wild-type receptor. These results raise the possibility that, in addition to altering downstream signaling pathways, the EGFR mutations may struc- turally alter the ATP pocket in such a way as to enhance binding by inhibitors and further increase the drug response.

\section{ACQUIRED RESISTANCE TO GEFITINIB: THE T790M MUTATION}

Rare patients with NSCLC harboring EGFR mutations may sustain prolonged clinical responses, but in most cases, an initial dramatic response to gefitinib is followed by tumor regrowth within about 6 months. Two groups have independently reported the presence of a secondary somatic EGFR mutation in such cases (Pao et al. 2004; Kobayashi et al. 2005), and to date, approximately 50\% of tumors with acquired drug resistance appear to harbor the same missense mutation, T790M. Remarkably, this mutation affects the homologous residue within the catalytic site of the ABL kinase domain targeted by the T315I mutation, frequently observed in imatinib-resistant CML. This so-called "gatekeeper" residue appears to modulate access of small-molecule inhibitors to the catalytic site. In our own studies, we also identified the T790M mutation in independent metastatic lesions, which had recurred after an initial response in two patients whose primary tumor harbored an activating EGFR mutation (Kwak et al. 2005). Surprisingly, however, the abundance of this drug resistance mutation was considerably lower than would be expected if it were the sole mechanism underlying acquired resistance. In the first patient, the wild-type and initial somatic activating EGFR mutation were present in a 1:1 ratio, as expected for a heterozygous mutation. However, the secondary drug resistance somatic mutation T790M was only observed at a 1:5 ratio to the wild-type allele. In the second case, where 4 independent liver metastases were analyzed, no T790M alleles were detected in an analysis of uncloned PCR products, but the mutation was detected at low levels in 2 of the 4 tumors following analysis of cloned PCR products (ranging in frequency from $1 / 55$ to 2/48 PCR-derived clones). Again, the first somatic mutation in all metastases was still present in the expected 1:1 ratio with the wild-type allele. No EGFR amplification was evident. We conclude that the T790M mutation is a "hot spot" for secondary mutations linked to acquired drug resistance but that other mechanisms are also likely to play a role in tumor recurrence. We note that even in cases with a dramatic response to gefitinib, only a partial response is achievable, and hence, the number of persistent tumor cells is very high. The acquisition of drug resistance in such cases is not a clonal process: We therefore hypothesize that a combination of mechanisms, some genetic (like T790M), others potentially epigenetic, may contribute to tumor regrowth following an initial response to gefitinib or erlotinib.

\section{ALTERED EGFR TRAFFICKING AND IN VITRO DRUG RESISTANCE}

To model the acquisition of resistance to gefitinib in NSCLC cells with activating EGFR mutations, we treated cell lines with the drug in vitro and selected multiple in- 
dependent drug-resistant clones (Kwak et al. 2005). The T790M mutation was not observed in this setting, nor was amplification of the mutant EGFR. However, 50- to 100fold resistance was readily achieved and appeared to be stable in the absence of drug selection. Analysis of receptor trafficking, using visualization of immunofluorescenttagged EGF in whole cells, as well as immunoblotting analysis of membrane-bound receptor using biotin-labeled ligand, revealed a consistent increase in receptor internalization following treatment with ligand. The mechanism underlying this alteration remains to be elucidated, and its clinical relevance cannot be readily ascertained in the absence of molecular markers that can be studied in clinical specimens. Nonetheless, these findings raise the possibility that epigenetic mechanisms may direct altered internalization of the receptor. Dissociation of gefitinib from the receptor is likely within the lower $\mathrm{pH}$ of endosomes, possibly leading to persistent downstream signaling. We note that gefitinib-resistant clones display persistent sensitivity to siRNA targeting EGFR, indicating that they remain "addicted" to EGFR signaling and appear not to have acquired additional genetic lesions in downstream effectors (Kwak et al. 2005).

\section{IRREVERSIBLE EGFR INHIBITORS: CIRCUMVENTING ACQUIRED DRUG RESISTANCE}

Both gefitinib and erlotinib are reversible inhibitors of EGFR that compete with ATP for binding to the catalytic pocket. Irreversible inhibitors of EGFR were initially developed to address potential pharmacodynamic concerns associated with competitive binding to the receptor. These agents, including EKB 569 (targeting EGFR) and HKI 272 (targeting both EGFR and erb-B2), made by Wyeth Pharmaceuticals, are similar in overall structure to the reversible inhibitors but differ in the presence of a so-called "Michael acceptor" which can form a covalent bond with Cys-773 within the catalytic pocket of the receptor (Rabindran et al. 2004). Our initial studies of these irreversible inhibitors indicated that they shared the selective killing of EGFR-mutant NSCLC cell lines and were comparable with reversible inhibitors in their relative effects on these cells versus those with wild-type EGFR. However, irreversible inhibitors demonstrated persistent activity against EGFR-mutant cells that had acquired resistance to gefitinib or erlotinib, either through expression of the secondary T790M mutation, or through apparent alterations in receptor internalization (Kwak et al. 2005). In both models, gefitinib was no longer effective in suppressing EGFR-mediated signaling or in cell killing, whereas the irreversible inhibitors showed persistent activity (Fig. 3). We presume that the improved reaction kinetics provided by the irreversible inhibition are sufficient to circumvent reduced drug binding due to the T790M mutation, as well as the potential dissociation of the drug-receptor complex resulting from altered internalization. Furthermore, we note that in marked contrast to reversible inhibitors, NSCLC cell lines with acquired resistance to irreversible inhibitors cannot be readily established in cell culture. Taken together, these observations have led to the initiation of clinical trials of HKI 272 in patients with EGFRmutant NSCLC that have acquired resistance to gefitinib and/or erlotinib. Should such trials prove encouraging, these "second-generation" EGFR inhibitors may warrant clinical testing in the initial treatment of patients with EGFR-mutant NSCLC.

Clinical studies are needed to test the effectiveness of irreversible EGFR kinase inhibitors predicted by in vitro studies. However, these observations raise the possibility that such covalent drug binding may prove important in targeting transmembrane growth factor receptor kinases, for which multiple mechanisms of drug resistance appear to be a common feature. Even for cytoplasmic kinases, such as ABL, the gatekeeper residue (codon 315 of BCR-
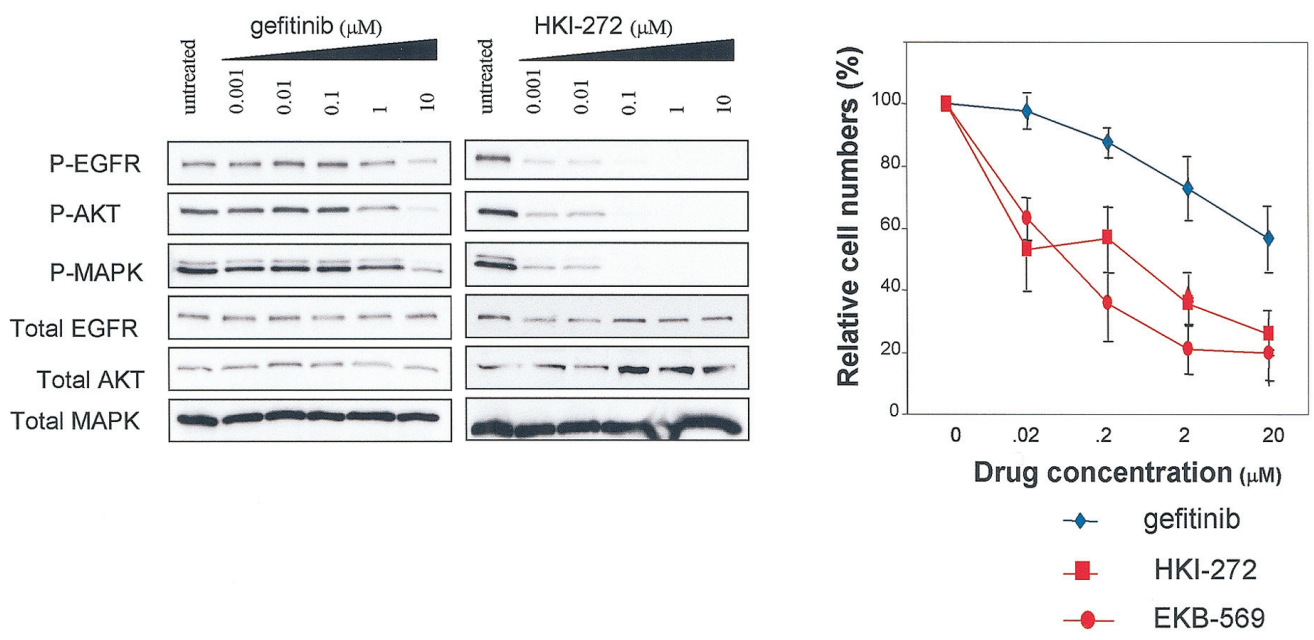

Figure 3. Suppression of EGFR autophosphorylation and AKT and MAPK phosphorylation by the irreversible inhibitor HKI 272 in cells that have an activating sensitizing EGFR mutation, but which have acquired resistance to gefitinib in culture. Failure of gefitinib to suppress EGFR, AKT, and MAPK phosphorylation is demonstrated (left panel). Cell killing induced by HKI 272 in these gefitinibresistant cell lines is shown (right panel). (Adapted from Kwak et al. 2005.) 
ABL) appears to be a hot spot for imatinib resistance, for which current second-generation ABL inhibitors targeting the open rather than closed configuration of the kinase have not proven effective (Shah et al. 2004). The effect of irreversible EGFR inhibitors in circumventing mutations affecting the analogous gatekeeper residue in EGFR offers hope that a similar strategy, if feasible, may hold some promise for refractory BCR-ABL mutations as well. By analogy with EGFR, the potential benefits of using irreversible inhibitors in targeting oncogenic kinases may not be evident initially, since both reversible and irreversible inhibitors may share comparable efficacy against previously untreated cells. As drug resistance emerges, however, irreversible inhibitors may prove capable of inducing longer-lasting responses. Whereas the toxicity profile of EKB 569 and HKI 272 appears minimal, this may not be true for other small molecules with such reactive side chains. Taken together, our in vitro observations raise the possibility that irreversible kinase inhibitors may offer advantages that need to be tested in clinical trials.

\section{CONCLUDING REMARKS}

Much remains to be understood with respect to targeting EGFR in NSCLC, but some lessons may be drawn, with potential implications for the treatment of lung cancer, as well as other cancers that may be targeted by "molecular therapeutics." In NSCLC, a relatively small number of somatic mutations capable of activating the kinase activity of EGFR are correlated with dramatic clinical responses to inhibitors of this pathway. Mutational analysis of EGFR is now commercially available, which will facilitate prospective genotype-directed studies of drug response. High-level EGFR amplification may also be associated with responsiveness to kinase inhibitors, and together with mutational activation, illustrates the importance of genetic alterations as molecular markers of drug response. Other genetic or epigenetic factors that modulate drug response are likely but remain to be identified. Despite their dramatic nature, the drug responses associated with EGFR-mutant tumors are unlikely to have a major effect in improving survival unless acquired drug resistance can be overcome. Ongoing clinical trials of irreversible EGFR inhibitors, which appear effective in vitro, may address this critical issue.

The concept that common epithelial malignancies may comprise multiple genetically defined subclasses, and that dramatic clinical responses to any given targeted therapy may be limited to a relatively small subset, is likely to have significant implications for the development and testing of these novel agents. The cost of earlyphase clinical trials may be significantly reduced if tumor genotyping can select a subset with a high probability of drug susceptibility. Reliable preclinical approaches to defining such drug-responsive tumor types need to be developed, and these may include a combination of nucleotide sequencing and in vitro functional analyses. The nature of early-phase studies themselves may evolve, as traditional dose escalation "Phase I toxicity studies" may become less relevant, given the minimal toxicity of these agents and the need to define appropriate dosing based on responsiveness in susceptible tumors, rather than limiting toxicity in nonresponsive cases. Although the potentially reduced costs of drug development associated with targeted clinical testing may be encouraging, concern has been raised about reduced financial incentives for pharmaceutical companies considering designing new drugs for smaller market shares. Nonetheless, the development of truly effective cancer therapies, as demonstrated by the success of Gleevec in the treatment of CML, provides an inspiring model for the application of targeted therapies in epithelial tumors.

\section{ACKNOWLEDGMENTS}

We are grateful to all the members of the Center for Molecular Therapeutics, Center for Cancer Risk Analysis, and Center for Thoracic Oncology of the Massachusetts General Hospital Cancer Center for their ongoing commitment and collaboration. This work was supported by grants from the National Institutes of Health, the Doris Duke Charitable Foundation, the National Foundation for Cancer Research, the Sandler Family Foundation, the V Foundation, the Saltonstall Foundation, and Sue's Fund for lung cancer research at Massachusetts General Hospital.

\section{REFERENCES}

Bell D.W., Lynch T.J., Haserlat S.M., Harris P.L., Okimoto R.A., Brannigan B.W., Sgroi D.C., Muir B., Riemenschneider M.J., Iacona R.B., Krebs A.D., Johnson D.H., Giaccone G., Herbst R.S., Manegold C., Fukuoka M., Kris M.G., Baselga J., Ochs J.S., and Haber D.A. 2005. Epidermal growth factor receptor mutations and gene amplification in non-small-cell lung cancer: Molecular analysis of the IDEAL/INTACT gefitinib trials. J. Clin. Oncol. 23: 8081.

Han S.W., Kim T.Y., Hwang P.G., Jeong S., Kim J., Choi I.S., Oh D.Y., Kim J.H., Kim D.W., Chung D.H., Im S.A., Kim Y.T., Lee J.S., Heo D.S., Bang Y.J., and Kim N.K. 2005. Predictive and prognostic impact of epidermal growth factor receptor mutation in non-small-cell lung cancer patients treated with gefitinib. J. Clin. Oncol. 23: 2493.

Hirsch F.R., Varella-Garcia M., McCoy J., West H., Xavier A.C., Gumerlock P., Bunn P.A., Jr., Franklin W.A., Crowley J., and Gandara D.R. (Southwest Oncology Group). 2005. Increased epidermal growth factor receptor gene copy number detected by fluorescence in situ hybridization associates with increased sensitivity to gefitinib in patients with bronchioloalveolar carcinoma subtypes: A Southwest Oncology Group Study. J. Clin. Oncol. 23: 6838.

Kobayashi S., Boggon T.J., Dayaram T., Janne P.A., Kocher O., Meyerson M., Johnson B.E., Eck M.J., Tenen D.G., and Halmos B. 2005. EGFR mutation and resistance of non-small-cell lung cancer to gefitinib. N. Engl. J. Med. 352: 786.

Kris M.G., Natale R.B., Herbst R.S., Lynch T.J., Jr., Prager D., Belani C.P., Schiller J.H., Kelly K., Spiridonidis H., Sandler A., Albain K.S., Cella D., Wolf M.K., Averbuch S.D., Ochs J.J., and Kay A.C. 2003. Efficacy of gefitinib, an inhibitor of the epidermal growth factor receptor tyrosine kinase, in symptomatic patients with non-small cell lung cancer: A randomized trial. J. Am. Med. Assoc. 290: 2149.

Kwak E.L., Sordella R., Bell D.W., Godin-Heymann N., Okimoto R.A., Brannigan B.W., Harris P.L., Driscoll D.R., Fidias P., Lynch T.J., Rabindran S.K., McGinnis J.P., Wissner A., Sharma S.V., Isselbacher K.J., Settleman J., and Haber D.A. 2005. Irreversible inhibitors of the EGF receptor may circum- 
vent acquired resistance to gefitinib. Proc. Natl. Acad. Sci. 102: 7665 .

Lynch T.J., Bell D.W., Sordella R., Gurubhagavatula S., Okimoto R.A., Brannigan B.W., Harris P.L., Haserlat S.M., Supko J.G., Haluska F.G., Louis D.N., Christiani D.C., Settleman J., and Haber D.A. 2004. Activating mutations in the epidermal growth factor receptor underlying responsiveness of non-small-cell lung cancer to gefitinib. N. Engl. J. Med. 350: 2129 .

Mellinghoff I.K., Wang M.Y., Vivanco I., Haas-Kogan D.A., Zhu S., Dia E.Q., Lu K.V., Yoshimoto K., Huang J.H., Chute D.J., Riggs B.L., Horvath S., Liau L.M., Cavenee W.K., Rao P.N., Beroukhim R., Peck T.C., Lee J.C., Sellers W.R., Stokoe D., Prados M., Cloughesy T.F., Sawyers C.L., and Mischel P.S. 2005. Molecular determinants of the response of glioblastomas to EGFR kinase inhibitors. N. Engl. J. Med. 353: 2012 .

Mitsudomi T., Kosaka T., Endoh H., Horio Y., Hida T., Mori S., Hatooka S., Shinoda M., Takahashi T., and Yatabe Y. 2005. Mutations of the epidermal growth factor receptor gene predict prolonged survival after gefitinib treatment in patients with non-small-cell lung cancer with postoperative recurrence. J. Clin. Oncol. 23: 2513.

Paez J.G., Janne P.A., Lee J.C., Tracy S., Greulich H., Gabriel S., Herman P., Kaye F.J., Lindeman N., Boggon T.J., Naoki K., Sasaki H., Fujii Y., Eck M.J., Sellers W.R., Johnson B.E., and Meyerson M. 2004. EGFR mutations in lung cancer: Correlation with clinical response to gefitinib therapy. Science 304: 1497.

Pao W., Miller V.A., Politi K.A., Riely G.J., Somwar R., Zakowski M.F., Kris M.G., and Varmus H. 2005. Acquired resistance of lung adenocarcinomas to gefitinib or erlotinib is associated with a second mutation in the EGFR kinase domain. PloS Med. 3: e73.

Pao W., Miller V., Zakowski M., Doherty J., Politi K., Sarkaria I., Singh B., Heelan R., Rusch V., Fulton L., Mardis E., Kupfer D., Wilson R., Kris M., and Varmus H. 2004. EGF receptor gene mutations are common in lung cancers from "never smokers" and are associated with sensitivity of tumors to gefitinib and erlotinib. Proc. Natl. Acad. Sci. 101: 13306.

Rabindran S.K., Discafani C.M., Rosfjord E.C., Baxter M., Floyd M.B., Golas J., Hallett W.A., Johnson B.D., Nilakantan R., Overbeek E., Reich M.F., Shen R., Shi X., Tsou H.R., Wang Y.F., and Wissner A. 2004. Antitumor activity of HKI272, an orally active, irreversible inhibitor of the HER-2 tyrosine kinase. Cancer Res. 64: 3958.

Riemenschneider M.J., Bell D.W., Haber D.A., and Louis D.N. 2005. Pulmonary adenocarcinomas with mutant epidermal growth factor receptors. N. Engl. J. Med. 352: 1724.

Shah N.P., Tran C., Lee F.Y., Chen P., Norris D., and Sawyers C.L. 2004. Overriding imatinib resistance with a novel ABL kinase inhibitor. Science 305: 399.

Sharma S.V., Fischbach M.A., Haber D.A., and Settleman J. 2006. Oncogenic shock: Explaining oncogene addiction through differential signal attenuation. Clin. Cancer Res. (in press).

Shepherd F.A., Rodrigues Pereira J., Ciuleanu T., Tan E.H., Hirsh V., Thongprasert S., Campos D., Maoleekoonpiroj S., Smylie M., Martins R., van Kooten M., Dediu M., Findlay B., Tu D., Johnston D., Bezjak A., Clark G., Santabarbara P., and Seymour L. (National Cancer Institute of Canada Clinical Trials Group). 2005. Erlotinib in previously treated non-smallcell lung cancer. N. Engl. J. Med. 353: 123.

Sordella R., Bell D.W., Haber D.A., and Settleman J. 2004 Gefitinib-sensitizing EGFR mutations in lung cancer activate anti-apoptotic pathways. Science 305: 1163.

Tsao M.S., Sakurada A., Cutz J.C., Zhu C.Q., Kamel-Reid S., Squire J., Lorimer I., Zhang T., Liu N., Daneshmand M., Marrano P., da Cunha Santos G., Lagarde A., Richardson F., Seymour L., Whitehead M., Ding K., Pater J., and Shepherd F.A. 2005. Erlotinib in lung cancer: Molecular and clinical predictors of outcome. N. Engl. J. Med. 353: 133.

Wakeling A.E., Guy S.P., Woodburn J.R., Ashton S.E., Curry B.J., Barker A.J., and Gibson K.H. 2002. ZD1839 (Iressa): An orally active inhibitor of epidermal growth factor signaling with potential for cancer therapy. Cancer Res. 62: 5749.

Weinstein I.B. 2002. Cancer. Addiction to oncogenes-The Achilles heal of cancer. Science 297: 63. 


\section{$8_{8}^{\infty} \mathrm{CSH} \&$ Cold Spring Harbor Symposia SYMPOSIA on Quantitative Biology}

\section{Molecular Targeted Therapy of Lung Cancer: EGFR Mutations and Response to EGFR Inhibitors}

D.A. HABER, D.W. BELL, R. SORDELLA, et al.

Cold Spring Harb Symp Quant Biol 2005 70: 419-426

Access the most recent version at doi:10.1101/sqb.2005.70.043

References This article cites 20 articles, 12 of which can be accessed free at: http://symposium.cshlp.org/content/70/419.full.html\#ref-list-1

License

Email Alerting Receive free email alerts when new articles cite this article - sign up in Service the box at the top right corner of the article or click here. 